\title{
The effect research of multimedia-assisted music teaching based on principal component analysis----- For example HeNan xuchang college
}

\author{
Li Ping \\ Xuchang University, Xuchang, Henan, China, 461000 \\ 360008910@qq.com
}

Keywords: Multimedia-assisted instruction; Teaching effect; Principal component analysis

\begin{abstract}
Currently, multimedia-assisted music instruction has been widely used in colleges and universities. The diversification trend of music teaching and greatly promoted students' absorbing ability have increased the extensive use of multimedia-assisted instruction in music teaching. To solve the problems like teachers rely too much on multimedia-assisted instruction in music teaching process, lack of effective interaction between teachers and students and lack of effectiveness, 126 pieces of music multimedia teaching effect questionnaires are analyzed and the music professional classes comprehensive scores of art major students of two sessions are compared. Principal component analysis method is applied to analyze the main factors influencing the music multimedia-assisted instruction effect. Conclusions have been drawn from the research that multimedia-assisted instruction can improve the absorbing ability of students' music learning, that teaching courseware of informative and interesting content may enhance students' interest in learning, that effective interaction between teachers and students can improve the effectiveness of multimedia music instruction, and that students accepting more multimedia-assisted music instruction turn to have better absorbing ability.
\end{abstract}

\section{Introduction}

In recent years, with the high-speed development of computer and network, multimedia-assisted music instruction has been widely used in colleges and universities. Multimedia teaching mode which combines with sound and photoelectric image technology has greatly enriched music culture's manifestation patterns, stimulated the absorbing ability of students' senses, improved the students' interest in learning music professional knowledge, and greatly improved teaching efficiency. Common multimedia teaching system is as shown in Figure 1.

With the rapid development of science and technology, music multimedia-assisted teaching is facing more opportunities and challenges and presents higher request for current music multimedia teaching. Educational concept, content and form must perform in-depth reform along with the progress of science and technology. Schools need to provide more interactive, intuitive and infectious multimedia-assisted music education teaching courses. More professional multimedia teaching method can further improve the quality and efficiency of music professional teaching, leave students more space and time for independent operations and activities, and cultivate high quality music talents with advanced modern music culture.

Although music multimedia-assisted teaching has advantages as comprehensive, objective, pertinent, large capacity and professional, and has been widely used in college music teaching, both the effectiveness and rationality of music multi-media teaching and teacher-dominance are problems which caused the scholars' attention. Lu Xiaoxu argues that at the same time of enriching music teaching patterns by applying multimedia teaching, problems like some music teachers rely too much on multimedia technology appear [1]. For the research of applying multimedia and network multimedia to ordinary university public music teaching, Li Fei has also pointed out that although multimedia teaching can mobilize students' learning interest and enthusiasm, multimedia technology may reverse the primary and secondary problems. Multimedia technology can not only help teachers better analyze and explain vocal music's synthesis and decomposition, but reduce the interaction 
between teachers and students in vocal music practice course, leading to teaching misunderstandings like lack of communication between teachers and students[2]. Dou Wenyu pointed out that in the course design of music specialized courses in normal universities, the application of multimedia technology should be cautiously applied in multi-voice music analysis and writing courses. Solfeggio teaching should combine the traditional teaching mode with modern multimedia teaching mode to avoid relying too much on multimedia technology and in the process of cultivating students' music skills, pay attention to train students' artistic awareness [3]. So a survey has been done to the students of music performing major and musicology major in one Shanghai university with questionnaires to study the impact of multimedia-assisted teaching on teaching effect.

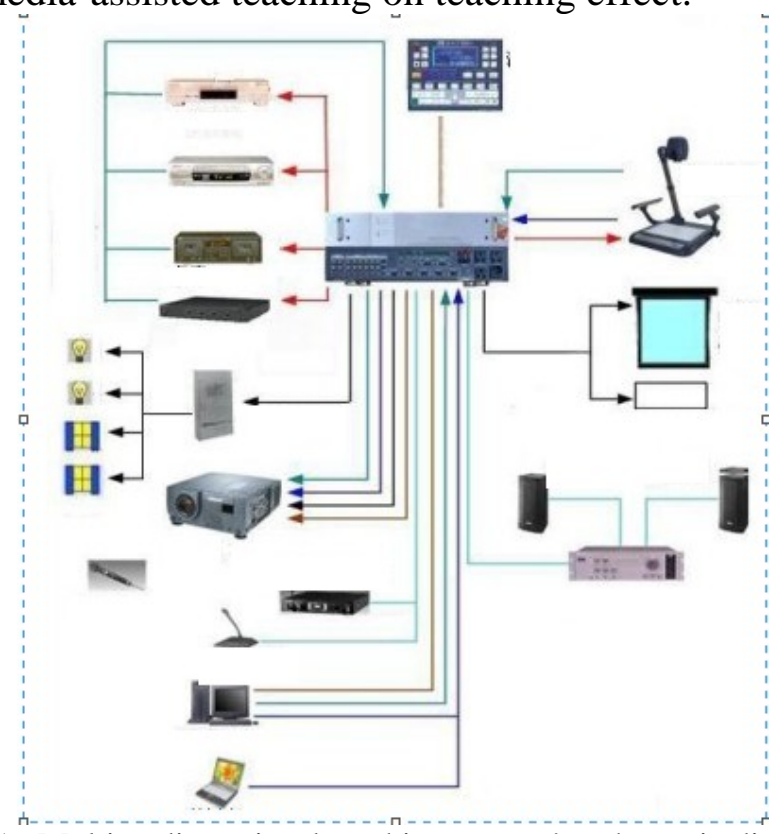

Fig 1. Multimedia-assisted teaching system's schematic diagram

\section{The main advantages of multimedia-assisted music teaching}

Multimedia teaching can be called computer assisted instruction (CAI), mainly refers to the teaching mode with the combination of a variety electronic media of computer hardware and software such as computer music, slides, and MIDI technology. Multimedia-assisted music teaching mode's appearance brought earth-shaking changes to the way and form of music teaching. The advantages of multimedia-assisted music teaching mainly lie in the following areas:

First of all, multimedia-assisted teaching strengthens the pluralism and intuitiveness of vocal music skills teaching means. Multimedia teaching enhances the interpretation of music skills practice teaching by demonstrating sound synthesis and decomposition. In music teaching, multimedia teaching, as the open auxiliary teaching model, breaks the traditional boring teaching methods. An electronic courseware can show students a variety of musical instruments' sounds at the same time, and show the diversity of sounds by adjusting sound frequency. Teachers' application of multimedia equipment can mobilize students' learning enthusiasm through a variety of forms of sensory stimulation and significantly promote music skills' teaching quality. The main advantage of multimedia teaching, therefore, is to overcome the problems of traditional teaching's single method, stiff teaching contents and so on. So, in leaping the boundaries of time and space, students may fully exert their imagination and creativity.

Second, multimedia-assisted music teaching can not only improve teaching efficiency by reducing the unnecessary time of teachers' teaching process, but simplify the teaching content. The teaching contents in multimedia-assisted music teaching are more simple and specific, which improves the efficiency of students' vocal music skills training. Multi-media music teaching process can still synthesize and discompose sounds by making music courseware of detailed software synthesis, elaborately show the comparison of a variety of different music styles, present the contents of 
electronic courseware from easy to difficult, and highlight the important content by applying volume change or other methods to deepen the students' memory. An electronic courseware may usually contain a large number of teaching contents, can cross the boundaries of time and space, perform transverse comparison, and strengthen the connection and communication among knowledge.

Although multimedia-assisted music teaching fully shows its strong creative and superiority, the widespread use of multimedia teaching puts forward higher requirements for teacher's computer hardware and software operation ability. Multimedia teaching requires teachers master relative advanced vocal operating knowledge of related software, and the ability to use computers and projectors to show the result made by the software to students. Some opponents have pointed out that teacher's computer multimedia teaching skills will significantly affect teaching effect. Famous professor Liu Huan adopts traditional teaching means in vocal music course teaching so far. With the emergence of self management multimedia teaching system, multimedia-assisted teaching is facing problems like technology upgrading and platform extensions. The fast rise in students number, the increasing students learning space requirements and the existing limited education resources construct more conflicts. Schools need to build a broader learning platform for students and produce more vivid and interesting coursewares in order to meet the needs of students.

\section{Multimedia-assisted music teaching research effect model}

130 questionnaires are handed out in the present study. Grade 2010 and 2011's music performing major and musicology major undergraduate students in a university in Shanghai are chosen to be the subjects. There are 130 effective questionnaires. Ruled out four students' questionnaires of failing grades and low credits, the practical application of questionnaires in the research is 126 . The annual comprehensive professional courses' scores of the subject students are taken as data sources, and principal component analysis is applied to study the effect of multimedia-assisted music teaching on the promotion of music professional undergraduate students' attainment.

Research hypothesis .Along with the rapid development of science and technology, knowledge transmission mode and dissemination platform have changed greatly. Network development and reform make students have more convenient access to learning resources, help students save time in collecting and screening data. Multimedia-assisted music teaching helps students to enjoy learning mode reform brought by the development of science and technology. Students can be more efficient in the application of computer software and hardware to absorb professional music knowledge. So hypothesis 1 is proposed.

Hypothesis 1: multimedia-assisted music teaching helps promote the effect of skill training for music professional students in colleges and universities.

A growing body of research argue that multimedia-assisted teaching can improve students' knowledge absorption capacity, but music teacher's professional level of computer software and hardware operation, including teachers' proficiency in multimedia equipment and projectors operation, and teachers' proficiency of pop music editing software will affect the teachers' application effect of multimedia-assisted teaching. Current professional music teachers' promotion rate of computer knowledge and application ability is often far below the development of computer science and technology. Teachers' operating level of multimedia teaching equipments may influence teaching effect. So hypothesis 2 is put forward.

Hypothesis 2: music professional teachers' computer software and hardware operation level will affect multimedia-assisted music teaching effect.

Music teaching is different from science and engineering, which needs cultivating students' skills and at the same time cultivate students' artistic accomplishment. Therefore the electronic courseware of multimedia-assisted music teaching should be appropriate, and has a certain artistic quality to improve the effect of music teaching. So hypothesis 3 is proposed.

Hypothesis 3: multimedia electronic courseware's design will affect multimedia-assisted music teaching effect.

Set research variables. 
1)Multimedia-assisted music teaching effect variables

Questionnaires of this study adopt real-name system in order to enhance comparability. The respondents of the study are music professional undergraduate students from a university in Shanghai. The students' music professional courses' comprehensive performance is taken as the dependent variable to measure multimedia-assisted music teaching effect, so as to enhance the authenticity of the results.

2)Multimedia-assisted music teaching's influencing factors variables

In this study, set the multimedia teaching hour students accepted x1, professional class teachers' average computer operation ability x2, electronic courseware's design level x3, and the advanced degree of multimedia teaching equipment $\mathrm{x} 4$ is taken as independent variables in order to enhance the credibility of the research.

3)Control variables

This article takes students characteristic variable as control variables, in which S1 is students' gender, and S2 is students' age.

Building research model. This paper used principal component analysis to study the effect of multimedia-assisted teaching in music teaching. The specific form of the model is as follows:

$\mathrm{y}_{i}=x_{i} \beta_{i}+\alpha_{i} S_{i}+\mu_{i}$ (Formula 3.1)

In it, $i=1,2, \ldots, N$.

In formula (3.1), the dependent variable is music professional students' comprehensive performance in 2012 academic year. $x_{i}$ is independent variable; $S_{i}$ is control variable; $\beta_{\mathrm{i}}$ and $\alpha_{\mathrm{i}}$ are respectively the coefficient of independent variables and control variables; $\mu_{\mathrm{i}}$ is independent random variable error term. The return form of Formula (3.1) is written in matrix form as:

$$
\left(\begin{array}{c}
\mathrm{y}_{1} \\
y_{2} \\
\vdots \\
y_{N}
\end{array}\right)=\left(\begin{array}{cccc}
x_{1} & 0 & \cdots & 0 \\
0 & x_{2} & \ddots & \vdots \\
\vdots & \ddots & \ddots & 0 \\
0 & \cdots & 0 & x_{N}
\end{array}\right)\left(\begin{array}{c}
\beta_{1} \\
\beta_{2} \\
\vdots \\
\beta_{N}
\end{array}\right)+\left(\begin{array}{c}
\mu_{1} \\
\mu_{2} \\
\vdots \\
\mu_{N}
\end{array}\right)+\left(\begin{array}{cccc}
s_{1} & 0 & \cdots & 0 \\
0 & s_{2} & \ddots & \vdots \\
\vdots & \ddots & \ddots & 0 \\
0 & \cdots & 0 & s_{N}
\end{array}\right)\left(\begin{array}{c}
\alpha_{1} \\
\alpha_{2} \\
\vdots \\
\alpha_{N}
\end{array}\right)
$$

And, $E\left(u_{i}\right)=0_{T \times 1} E\left(u_{i} u_{i}^{\prime}\right)=\sigma_{u}^{2} I_{T} \quad E\left(u_{i} u_{j}^{\prime}\right)=0_{T \times T}(i \neq j)$,in which $\mathrm{I}=1,2, \ldots$, IT is the dimension unit matrix of $\mathrm{T} \times \mathrm{T}$.

Parameters $\alpha_{i}$ and 's optimal estimationns are calculated by means of least square method, and the formula is:

$$
\begin{aligned}
& \hat{\beta}=\left[\sum \sum\left(x_{i t}-\bar{x}_{i}\right)\left(x_{i t}-\bar{x}_{i}\right)^{\prime}\right]^{-1}\left[\sum \sum\left(x_{i t}-\bar{x}_{i}\right)\left(y_{i t}-\bar{y}_{i}\right)\right] \\
& \hat{\alpha}_{i}=\overline{y_{i}}-\bar{x}_{i} \hat{\beta}
\end{aligned}
$$

The calculation formula of dependent variable residual error is as follows:

$$
R S S=\sum_{i=1}^{N} \hat{u_{i}^{2}}=\sum_{i=1}^{N}\left(y_{i}-\hat{y_{i}}\right)^{2}=\sum_{i=1}^{N}\left(\mathrm{y}_{i}-a_{0}-a_{i} x_{i}\right)^{2}
$$

\section{Empirical research results of multimedia-assisted music teaching effect}

\section{Descriptive statistical analysis}

Tab 1. Descriptive statistics

\begin{tabular}{|c|c|c|c|c|}
\hline & Min & Max & Mean & Std. Deviation \\
\hline X1 & 1.44 & 2.06 & 1.97 & 10.47 \\
\hline X2 & 1.05 & 4.24 & 3.14 & 15.72 \\
\hline
\end{tabular}




\begin{tabular}{|c|c|c|c|c|}
\hline $\mathrm{X} 3$ & 1.12 & 1.98 & 1.53 & 5.03 \\
\hline $\mathrm{X} 4$ & 1.02 & 1.64 & 1.32 & 4.38 \\
\hline $\mathrm{S} 1$ & 0.80 & 0.8216 & 0.81 & 3.59 \\
\hline $\mathrm{S} 2$ & 0.98 & 3.05 & 2.05 & 4.57 \\
\hline
\end{tabular}

After descriptive statistics analysis of the 126 questionnaires data, descriptive statistics results of the sample are got. According to the results, first, students of 2011 grade have more multimedia-assisted teaching courses than those of 2010 grade, so the students' professional class scores compare results of two grades can better explain the impact of the multimedia-assisted music teaching. Second, students' subjective judgments of the teachers' computer application ability are largely different, which means that the professional familiarity of related equipments for multimedia teaching between music teachers is different, and its impact on teaching effect also needs studying. Third, although students are from different music majors of the same college, the number difference of boys and girls is not big. Fourth, students' subjective impression of the complexity of electronic courseware and multimedia equipments' advanced degree are similar. The student generally accept that electronic coursewares are easy to understand, and because the students' grades are close, their impression of the school's multimedia equipment update progress is not obvious.

The empirical research results of multimedia-assisted music teaching effect

Tab 2. Principal component analysis' study results

\begin{tabular}{|c|c|c|c|c|}
\hline \multicolumn{5}{|c|}{ Dependent Variable: Y } \\
\hline \multicolumn{5}{|c|}{ Included observations: 155 } \\
\hline Variable & Coefficient & Std. Error & t-Statistic & Prob. \\
\hline C & 2.1414 & 7.2979 & 0.9003 & 0.0317 \\
\hline X1 & 12.4327 & 0.7193 & 2.5427 & 0.0264 \\
\hline X2 & 15.7612 & 1.1515 & 2.5773 & 0.0433 \\
\hline X3 & -1.8854 & 1.4143 & -1.1254 & 0.2325 \\
\hline X4 & 2.7919 & 1.0562 & 0.8916 & 0.1341 \\
\hline S1 & 0.9226 & 0.5186 & 0.7382 & 0.0512 \\
\hline S2 & 1.6032 & 0.9374 & 1.2096 & 0.7129 \\
\hline
\end{tabular}

Principal component analysis is applied in this article to study the music teaching effect of multimedia-assisted teaching. It can be seen from Table II that the greater is multimedia-assisted music lesson hours $\mathrm{x} 1$, the better teachers' multimedia equipment operation ability $\mathrm{x} 2$. The more advanced is multimedia teaching equipment $\mathrm{x} 3$, the higher are students' professional class comprehensive scores, that is, the more obvious is the effect of multimedia-assisted music teaching. The variables' coefficients $\mathrm{x} 1$ and $\mathrm{x} 2$ are larger and their $\mathrm{P}$ values are less than 0.05 , which shows that multimedia-assisted music lesson hours and teachers multimedia equipments' operation ability have obvious promoting effect on music teaching, and have significant statistical significance. Although multimedia equipments' advanced degree will affect teaching effect, but the impact is not obvious. In addition, as can be seen from Table 4-2, the coursewares' complexity may have side effects on teaching effect. The main cause is perhaps that the electronic courseware is too easy to have better promotion of teaching effect. If teachers increase the difficulty of electronic courseware, multimedia-assisted teaching effect may be improved. Students' gender control variables have little effect on teaching effect, that means regardless of their gender, students absorptive capacities in multimedia-assisted teaching are similar, while the older students' accepting ability is slightly better.

Through the analysis of Table II, we can draw the following conclusions: first, multimedia-assisted music teaching will enhance the student's professional courses' grades, namely multimedia-assisted teaching may enhance students' skill training effect, therefore hypothesis 1 is true. The reason for this is that multimedia-assisted music teaching shows more different forms of music culture. Multimedia devices can more comprehensively display the content and form of music skills training, therefore, multimedia teaching can help students absorb music skills training courses.

Second, music culture's diversification process is accelerating, music professional teachers' operation ability of multimedia equipment will influence the courses' rendering effects, thus to 
influence students' absorption capacity. Therefore hypothesis 2 is true. At the same time, because of the limited computer and multimedia operation ability of music professional teachers, it is hard to make perfect electronic courseware like professional computer operators. Currently most of the music curriculum's electronic coursewares are still in the initial design stage. With a few network reference resources, music professional teachers' electronic coursewares are relatively easy, and have limited effects on students' absorption. So hypothesis 3 is false. More feasible method to solve this problem is to build network teaching platform by absorbing more music major related people to join the making of electronic courseware content and appropriately hiring computer professionals to further improve the present form of electronic courseware.

\section{Conclusion}

Diversified development trend of contemporary music and the demand of professional students for professional knowledge have exceeded. With rapid development of science and technology, the wide application of multimedia technology can promote more music forms and music culture. Music professional education of higher learning need to deepen the reform. The traditional solfeggio and ear training teaching teaching mode has the need for appropriate technology updates. Multimedia-assisted teaching has become the indispensable auxiliary teaching way for new century education. Colleges and universities should think deeply about music attainments' cultivation mode and avoid blind worship of the multimedia teaching mode or computer teaching one-sidedly in the use of multimedia-assisted music teaching. Under the present circumstances, colleges and universities should further study how music education teaching itself may meet the demand of the new knowledge era, and provide broader learning platform for students by breaking through cultural restrictions. This is the real meaning of applying multimedia-assisted instruction in teaching.

\section{References}

[1] Lu Xiaoxu, Jiang Zhenxing. Computer music technology and practice [M]. Tsinghua University Press, 2005.

[2] Li Fei. A preliminary study of advantages and problems in the application of multimedia and network multimedia in ordinary university public music teaching [D]. Capital Normal University, 2006.

[3] Dou Wenyu. Multimedia technology’s course application study in college music professional "Multi-tone Music Analysis and Writing" Xinjiang Normal University, 2009.

[4] Luo Hongmin. Analyses of the problems in the application of multimedia technology in music education [J]. Big Stage, 2010 (6).

[5] Wang Chenglai. Effective application of multimedia in the teaching of solfeggio and ear training teaching [D]. Xi 'an Music College, 2010.

[6] zhang Jing. The application of modern multimedia technology in music teaching [J]. Comprehensive Digest (management), 2009 (9). 